\title{
FONCTIONS SPHÉRIQUES DES ESPACES SYMÉTRIQUES COMPACTS
}

\author{
JEAN-LOUIS CLERC
}

\begin{abstract}
An integral formula, similar to Harish-Chandra's formula for spherical functions on a noncompact Riemannian symmetric space $G / K$ is given for the spherical functions of the compact dual $U / K$. As a consequence, an asymptotic expansion, as the parameter tends to infinity, is obtained, by using the (complex) stationary phase method.
\end{abstract}

RÉSUMÉ. On démontre une formule intégrale pour les fonctions sphériques d'un espace symétrique de type compact $U / K$, analogue de la formule d'HarishChandra pour le dual non-compact $G / K$. En conséquence on obtient un équivalent asymptotique lorsque le paramètre tend vers l'infini, en utilisant la méthode de la phase stationnaire complexe.

Les fonctions sphériques des espaces symétriques compacts sont nettement moins bien étudiées que leurs homologues du cas non-compact; on se propose dans cet article de généraliser deux résultats classiques sur les polynômes de Legendre, qui correspondent aux fonctions sphériques de l'espace $S O(3) / S O(2)$ : d'une part, la représentation intégrale, dite de première espèce et due à Laplace

$$
P_{n}(\cos \theta)=\frac{1}{2 \pi} \int_{0}^{2 \pi}(\cos \theta+i \sin \theta \cos \varphi)^{n} d \varphi
$$

d'autre part l'équivalent asymptotique, du à Laplace et Heine

$$
0<\theta<\pi, \quad P_{n}(\cos \theta) \sim \sqrt{\frac{2}{\pi n}} \frac{\cos \left[\left(n+\frac{1}{2}\right) \theta-\pi / 4\right]}{\sqrt{\sin \theta}}, \quad n \rightarrow+\infty .
$$

Voir [12] pour ces résultats élémentaires.

1. Complexification des décompositions classiques. Soit $\mathfrak{g}$ une algèbre de Lie semi-simple réelle, de type non-compact, $\theta$ une involution de Cartan de $\mathfrak{g}$, à laquelle correspond la décomposition $\mathfrak{g}=\mathfrak{k} \oplus \mathfrak{p}$. Soit $\mathfrak{a} \subset \mathfrak{p}$ un sous-espace de Cartan, $\Sigma$ le système des racines (restreintes) de $(\mathfrak{g}, \mathfrak{a})$, et $\Sigma^{+}$les racines positives pour le choix d'un ordre sur $\mathfrak{a}^{*}$. Si $\lambda \in \Sigma$, on note $\mathfrak{g}_{\lambda}$ le sous-espace radiciel associé, et on pose

$$
\mathfrak{n}=\sum_{\lambda \in \Sigma^{+}} \mathfrak{g}_{\lambda}, \quad \overline{\mathfrak{n}}=\theta(\mathfrak{n})=\sum_{\lambda \in \Sigma^{+}} \mathfrak{g}_{-\lambda}
$$

On désigne par $\mathfrak{m}$ le centralisateur de $\mathfrak{a}$ dans $\mathfrak{k}$.

Soit $\mathfrak{g}_{\mathbf{C}}$ la complexifiée de $\mathfrak{g}$. Si $\mathfrak{z}$ est une sous-algèbre de $\mathfrak{g}$, on note $\mathfrak{z} \mathbf{C}$ sa complexifiée, regardée comme une sous-algèbre de $\mathfrak{g}_{C}$.

Received by the editors November 18, 1986.

1980 Mathematics Subject Classification (1985 Revision). Primary 43A90; Secondary 22E46. 
Soit encore $\mathfrak{u}=\mathfrak{k}+i \mathfrak{p} ;$ c'est une forme réelle compacte de $\mathfrak{g}_{\mathbf{C}}$.

Soit $G_{\mathbf{C}}$ le groupe de Lie complexe simplement connexe d'algèbre de Lie $g_{\mathbf{C}}$, et soit $G, K, A, N, \bar{N}, K_{\mathbf{C}}, A_{\mathbf{C}}, N_{\mathbf{C}}, \bar{N}_{\mathbf{C}}, U$ les sous-groupes analytiques correspondant aux sous-algèbres introduites précédemment. L'involution $\theta$ s'étend en une involution de $\mathfrak{g}_{\mathbf{C}}$, et celle-ci est la différentielle d'une involution de $G_{\mathbf{C}}$, notée encore $\theta$. Les points fixes de cette involution dans $G_{\mathbf{C}}$ forment un sous-groupe connexe de $G_{\mathbf{C}}$, d'algèbre de Lie $\mathfrak{k}_{\mathbf{C}}$, donc cö̈ncident avec $K_{\mathbf{C}}$. De même comme $U$ est un sous-groupe compact maximal de $G_{\mathbf{C}}$, il est simplement connexe, et les points fixes de $\theta$ dans $U$ forment un sous-groupe connexe, d'algèbre de Lie $\mathfrak{k}$, donc coïncident avec $K$.

Soit $\mathfrak{h}$ une sous-algèbre de Cartan de $\mathfrak{g}$ contenant $\mathfrak{a} ; \mathfrak{h}$ est $\theta$-stable. Soit $\Delta$ le système des racines de la paire $\left(\mathfrak{g}_{\mathbf{C}}, \mathfrak{h}_{\mathbf{C}}\right)$, et soit $\psi=\left\{\alpha \in \Delta,\left.\alpha\right|_{\mathfrak{a}_{\mathbf{C}}}=0\right.$ ou $\left.\left.\alpha\right|_{\mathfrak{a}} \in \Sigma^{+}\right\}$.

La partie $\psi$ est parabolique, et donc $\mathfrak{q}_{\mathbf{C}}=\mathfrak{h}_{\mathbf{C}}+\sum_{\alpha \in \psi}\left(\mathfrak{g}_{\mathbf{C}}\right)_{\alpha}$ est une sous-algèbre parabolique. Si $\left.\alpha\right|_{a_{C}}=0$, alors le sous-espace radiciel correspondant est contenu dans $\mathfrak{k}_{\mathbf{C}}$, et donc aussi dans $\mathfrak{m}_{\mathbf{C}}$. On en déduit aisément que $\mathfrak{q}_{\mathbf{C}}=\mathfrak{m}_{\mathbf{C}} \oplus \mathfrak{a}_{\mathbf{C}} \oplus \mathfrak{n}_{\mathbf{C}}$.

Soit $Q_{\mathrm{C}}$ le sous-groupe parabolique (fermé et connexe) correspondant.

(1.1) Proposition. L'application $(\bar{n}, \bar{q}) \rightarrow \bar{n} q \bar{N}_{\mathbf{C}} \times Q_{\mathbf{C}} \rightarrow G_{\mathbf{C}}$ est une submersion injective sur un ouvert de $G_{\mathbf{C}}$.

Comme $\mathfrak{g}_{\mathbf{C}}=\overline{\mathfrak{n}}_{\mathbf{C}} \oplus \mathfrak{q}_{\mathbf{C}}$, l'application est une submersion sur un ouvert de $\boldsymbol{F}_{\mathbf{C}}$. Reste à voir que $\bar{N}_{\mathbf{C}} \cap Q_{\mathbf{C}}$ est réduit à l'élément neutre ce qui entraînera l'injectivité. Soit $H$ un élément régulier de $\mathfrak{a}$; alors si $\bar{n} \in \bar{N}_{\mathbf{C}} \cap Q_{\mathbf{C}}$, on a $\operatorname{Ad} \bar{n}\left(\mathfrak{n}_{\mathbf{C}}\right) \subset \mathfrak{n}_{\mathbf{C}}$, puisque $Q_{\mathbf{C}}$ n'est autre que le normalisateur de $\mathfrak{n}_{\mathbf{C}}$, par suite, pour tout $X \in \mathfrak{n}_{\mathbf{C}}$, on a $B(\operatorname{Ad} \bar{n} \cdot X, H)=0$, puisque $\mathfrak{n}_{\mathbf{C}}$ et $\mathfrak{a}_{\mathbf{C}}$ sont orthogonaux pour la forme de Killing. Ou encore $B\left(X, \operatorname{Ad} \bar{n}^{-1} \cdot H\right)=0$, pour tout $X \in \mathfrak{n}_{\mathbf{C}}$. Mais $\operatorname{Ad} \bar{n}^{-1} H-H \in \overline{\mathfrak{n}}_{\mathbf{C}} ;$ comme $B(X, H)=0$ pour tout $X \in \mathrm{n}_{\mathbf{C}}$, et que $B$ est une dualité non-dégénérée entre $\overline{\mathfrak{n}}_{\mathbf{C}}$ et $\mathfrak{n}_{\mathbf{C}}$, on en déduit que $\operatorname{Ad} \bar{n}^{-1} H-H=0$; mais pour $H$ régulier, l'application $n \rightarrow \operatorname{Ad} n \cdot H-H$ est bijective (démonstration analogue au cas réel). Par suite $\bar{n}=e$.

Soit $\mathfrak{m}_{1}=\mathfrak{m} \oplus \mathfrak{a}$ le centralisateur de $\mathfrak{a}$ dans $\mathfrak{g}$, et $\left(M_{1}\right)_{\mathbf{C}}$ le centralisateur de $\mathfrak{a}_{\mathbf{C}}$ dans $G_{\mathbf{C}}$; on sait que $\left(M_{1}\right)_{\mathbf{C}}$ est un sous-groupe de Lie connexe, d'algèbre de Lie $\left(\mathfrak{m}_{1}\right)_{\mathbf{C}}$, et qu'il normalise $\mathfrak{n}_{\mathbf{C}}$. D'autre part il est élémentaire que $\left(M_{1}\right)_{\mathbf{C}} \cap N_{\mathbf{C}}=\{e\}$.

(1.2) Proposition. L'application $\left(M_{1}\right) \times N_{\mathbf{C}} \rightarrow Q_{\mathbf{C}}\left(m_{1}, n\right) \rightarrow m_{1} n$ est un homomorphisme bijectif de groupes de Lie.

Soit $M_{\mathbf{C}}$ le centralisateur de $a_{\mathbf{C}}$ dans $K_{\mathbf{C}} ; M_{\mathbf{C}}$ n'est pas en général connexe. Soit $F=\left\{a \in A_{\mathbf{C}}, a^{2}=e\right\}$.

(1.3) Proposition. (i) $M_{\mathbf{C}}=F \cdot\left(M_{\mathbf{C}}\right)_{0}$,

(ii) $M_{\mathbf{C}} \cap A_{\mathbf{C}}=F$.

DÉmonstration DE (i). Si $a \in F$, alors comme $a \in A_{\mathrm{C}}$, on a $\theta(a)=a^{-1}$; d'où $\theta(a)=a$, et donc $F \subset K_{\mathbf{C}}$, d'où on déduit $F \cdot\left(M_{\mathbf{C}}\right)_{0} \subset M_{\mathbf{C}}$.

Réciproquement, comme $M_{\mathbf{C}}$ est clairement un groupe réductif, on a $M_{\mathbf{C}}=$ $\left(M_{\mathbf{C}} \cap U\right) \exp \left(i \mathrm{u} \cap \mathrm{m}_{\mathbf{C}}\right)$.

Si $m \in M_{\mathbf{C}} \cap U$, alors $m$ commute au tore $\exp i \mathfrak{a}$; donc il existe un tore maximal $T$ de $U$ contenant à la fois $m$ et $\exp i \mathfrak{a}$. Son algèbre de Lie $\mathfrak{t}$ est $\theta$-stable, $\mathfrak{t} \cap i \mathfrak{p}=i \mathfrak{a}$, et $\mathfrak{t} \cap \mathfrak{k} \subset \mathfrak{m}$. De sorte qu'il existe $Z \in \mathfrak{m}$ et $H \in \mathfrak{a}$, tels que $m=\exp Z \exp i H$. 
Mais $\theta(m)=m$ implique $m=\exp Z \exp (-i H)$; d'où $\exp 2 i H=e$. Autrement dit $\exp i H \in F$; ceci prouve (i).

Pour (ii), on a $F \subset M_{\mathbf{C}} \cap A_{\mathbf{C}}$; si maintenant $a \in A_{\mathbf{C}}$, on a $\theta(a)=a^{-1}$, et donc $a \in M_{\mathbf{C}}$ implique $\theta(a)=a$; d'où $a^{2}=e$. On a en fait le résultat plus fort: $K_{\mathbf{C}} \cap A_{\mathbf{C}}=F$.

On peut donner de $F$ une description plus explicite (cf. [6, p. 322]). Pour cela introduisons pour chaque $\lambda \in \Sigma$ l'élément $A_{\lambda} \in \mathfrak{a}$, défini par $B\left(H, A_{\lambda}\right)=\lambda(H)$, pour tout $H \in \mathfrak{a}$.

(1.4) Proposition. Soit $L=\{H \in \mathfrak{a} \mid \exp 2 i H=e\}$; alors $L$ est le réseau engendré par les vecteurs $2 \pi A_{\lambda} /(\lambda, \lambda), \lambda \in \Sigma$.

Il est désirable - et nécessaire - de préciser dans la suite l'ouvert de la proposition (1.1). Pour ce faire, considérons une représentation irréducible $\pi$, de dimension finie et de classe 1 de la paire $(G, K)$. Celles-ci sont exactement les représentations holomorphes de dimension finie de $g_{C}$ admettant un vecteur $k$-fixe; d'après [7] elles sont caractérisées par leurs poids dominant. Plus précisément, fixons un ordre sur $\mathfrak{h}^{*}$ compatible avec celui fixé sur $\mathfrak{a}^{*}$ (c'est-à-dire tel que $\left.\mu\right|_{\mathfrak{a}}>0$ implique $\mu>0$ ). On a alors la caractérisation suivante.

(1.5) Proposition. Soit $\pi$ une représentation de classe 1 de la paire $(G, K)$. Le poids dominant correspondant $\mu \in \mathfrak{h}^{*}$ satisfait les conditions

(i) $\left.\mu\right|_{\mathfrak{h}_{\mathbf{c}} \cap \mathfrak{t}_{\mathbf{c}}}=\mathbf{0}$,

(ii) $(\mu, \lambda) /(\lambda, \lambda) \in \mathbf{Z}^{+}$, pour tout $\lambda \in \Sigma^{+}$. Inversement si $\mu \in \mathfrak{a}^{*}$ satisfait la condition

(iii) $(\mu, \lambda) /(\lambda, \lambda) \in \mathbf{Z}^{+}$, pour tout $\lambda \in \Sigma^{+}$, alors $\mu$ prolongée par 0 sur $\mathfrak{h}_{\mathbf{C}} \cap \mathfrak{k}_{\mathbf{C}}$ est le poids dominant d'une représentation de classe 1 de la paire $(G, K)$.

On note $\Lambda$ le sous-ensemble de $\mathfrak{a}^{*}$ formé des formes linéaires satisfaisant la condition (iii).

Pour $\mu \in \Lambda$, on note $\pi_{\mu}$ la représentation de $G_{C}$ associée; soit $\chi_{\mu}$ le caractère

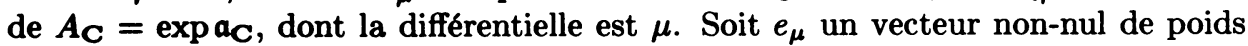
$\mu$. Alors les propositions (1.2), (1.3) et (1.4) permettent d'établir que $e_{\mu}$ est en fait invariant par $M_{\mathbf{C}} \cdot N_{\mathbf{C}}$ (cf. [7, p. 536]). On a donc $\pi\left(\right.$ man) $e_{\mu}=\chi_{\mu}(a) e_{\mu}$, pour $m \in M_{\mathbf{C}}, a \in A_{\mathbf{C}}$ et $n \in N_{\mathbf{C}}$. On note encore $\chi_{\mu}$ le caractère de $Q_{\mathbf{C}}$ trivial sur $M_{\mathbf{C}}$ et $N_{\mathbf{C}}$ et prolongeant $\chi_{\mu}$ sur $A_{\mathbf{C}}$.

Soit $E_{\mu}$ l'espace vectoriel de la représentation $\pi_{\mu}$, et munissons $E_{\mu}$ d'un produit scalaire hilbertien invariant par la restriction de $\pi_{\mu}$ à $U$, et choisissons $e_{\mu}$ de norme 1. On a alors, pour $\bar{n} \in \bar{N}_{\mathrm{C}}$ et $q \in Q_{\mathrm{C}}$

$$
\left\langle\pi_{\mu}(\bar{n} q) e_{\mu}, e_{\mu}\right\rangle=\chi_{\mu}(q) .
$$

En effet $\pi_{\mu}(q) e_{\mu}=\chi_{\mu}(q) e_{\mu}$, et $\pi_{\mu}(\bar{n}) e_{\mu}=e_{\mu}$ modulo une somme de vecteurs dans des sous-espaces de poids strictement plus petit que $e_{\mu}$, et donc orthogonaux à $e_{\mu}$. D'où l'égalité.

Posons $F_{\mu}(g)=\left\langle\pi_{\mu}(g) e_{\mu}, e_{\mu}\right\rangle$, et soit $\Omega_{\mu}=\left\{g \in G_{\mathbf{C}}, F_{\mu}(g) \neq 0\right\}$. Clairement $\Omega_{\mu}$ est un ouvert dense connexe de $G_{\mathbf{C}}$. 
(1.6) THÉORÈme. Soit $\bar{n} \in \bar{N}_{\mathbf{C}}$ et $q \in Q_{\mathbf{C}}$; alors $\bar{n} q \in \Omega_{\mu}$; si de plus $\mu$ est régulier, alors si $g \in \Omega_{\mu}$, il existe un unique $\bar{n} \in \bar{N}_{\mathbf{C}}$ et un unique $q \in Q_{\mathbf{C}}$, tels que $g=\bar{n} q$. En particulier $\Omega_{\mu}$ ne dépend pas de $\mu$, et l'application $g \rightarrow(\bar{n}, q)$ $\Omega_{\mu} \rightarrow \bar{N}_{\mathrm{C}} \times Q_{\mathrm{C}}$ est un difféomorphisme analytique.

Soit $\Omega$ l'image de l'application $(\bar{n}, q) \rightarrow \bar{n} q\left(\bar{n} \in \bar{N}_{\mathbf{C}}, q \in Q_{\mathbf{C}}\right)$. Nous savons que c'est un ouvert, et que $\Omega \subset \Omega_{\mu}$. Comme $\Omega_{\mu}$ est connexe, il nous suffit de démontrer que $\Omega$ est fermé relativement à $\Omega_{\mu}$. Soit donc $\left(g_{i}\right)$ une suite d'éléments de $\Omega$ convergeant vers un élément $g$ de $\Omega_{\mu}$.

On a $g_{i}=\bar{n}_{i} q_{i}$, et $F_{\mu}\left(g_{i}\right)=\chi_{\mu}\left(q_{i}\right) \rightarrow F_{\mu}(g) \neq 0$.

Par suite:

$$
\pi_{\mu}\left(\bar{n}_{i}\right) e=\pi_{\mu}\left(g_{i} q_{i}^{-1}\right) e_{\mu}=\chi_{\mu}\left(q_{i}\right)^{-1} \pi_{\mu}\left(g_{i}\right) e_{\mu}
$$

a pour limite $F_{\mu}(g)^{-1} \pi_{\mu}(g) e_{\mu}$.

(1.7) LEMME. Soit $\bar{n}_{i}$ une suite dans $\bar{N}_{\mathbf{C}}$, telle que $\pi\left(\bar{n}_{i}\right) e_{\mu}$ converge dans $E_{\mu}$; alors la suite $\left(\bar{n}_{i}\right)$ est convergente.

C'est une variante du lemme 40 de [5]; la démonstration ne réclame que des modifications mineures laissées au lecteur. L'hypothèse que $\mu$ est régulier est essentielle ici.

De ce lemme on déduit aussitôt que $\left(\bar{n}_{i}\right)$ est une suite convergente, disons vers $\bar{n} \in \bar{N}_{\mathrm{C}}$. Par suite $q_{i}=\left(\bar{n}_{i}\right)^{-1} g_{i}$ converge vers $(\bar{n})^{-1} g$. Mais comme $Q_{\mathrm{C}}$ est fermé, il s'ensuit que $(\bar{n})^{-1} g=q \in Q_{\mathrm{C}}$. D'où le résultat.

Le reste de la démonstration du théorème est élémentaire.

(1.8) THÉORÈME. L'application

$$
\bar{N}_{\mathbf{C}} \times\left(M_{1}\right)_{\mathbf{C}} \times N_{\mathbf{C}} \rightarrow G_{\mathbf{C}}
$$

donnée par $\left(\bar{n}, m_{1}, n\right) \rightarrow \bar{n} m_{1} n$ est un difféomorphisme analytique sur l'ouvert $\Omega$.

C'est une conséquence du théorème (1.6) et de la proposition (1.2). Abordons mintenant la complexification de la décomposition d'Iwasawa.

(1.9) Proposition. (i) L'application $(k, a, n) \rightarrow$ kan est une submersion de $K_{\mathbf{C}} \times A_{\mathbf{C}} \times N_{\mathbf{C}}$ sur un ouvert $\omega$ de $G_{\mathbf{C}}$.

(ii) $K_{\mathrm{C}} \cap A_{\mathrm{C}} N_{\mathrm{C}}=F$.

La partie (i) est conséquence de l'égalité $\mathfrak{g}_{\mathbf{C}}=\mathfrak{k}_{\mathbf{C}} \oplus \mathfrak{a}_{\mathbf{C}} \oplus \mathfrak{n}_{\mathbf{C}}$.

Pour (ii), il est clair que $F$ est contenu dans $K_{\mathbf{C}} \cap A_{\mathbf{C}} N_{\mathbf{C}}$.

Si $a \in A_{\mathbf{C}}$ et $n \in N_{\mathbf{C}}$, on a $\theta(a n)=a^{-1} \theta(n)$, et donc si $a n \in K_{\mathbf{C}}$, on a $a^{-1} \theta(n)=a n ;$ d'où $a^{2}=\theta(n) n^{-1} ;$ d'après la proposition (1.1) cela implique $a^{2}=e$, et donc $n=e$; d'où le résultat.

On va donner de l'ouvert $\omega$ une caractérisation analogue à celle du théorème (1.6).

Pour chaque point dominant $\mu$ d'une représentation de classe 1 , soit $e_{K}$ un vecteur $K$-fixe non nul (on ne fait pas figurer explicitement la dépendance en $\mu$ ). Alors $\left\langle e_{K}, e_{\mu}\right\rangle \neq 0$ (cf. [7, p. 537]). On peut donc supposer que ce produit scalaire vaut 1 .

Soit $f_{\mu}$ la fonction sur $G_{\mathbf{C}}$ définie par

$$
f_{\mu}(g)=\left\langle\pi(g) e_{\mu}, e_{K}\right\rangle .
$$


Soit $\omega_{\mu}=\left\{g \in G_{\mathbf{C}}, f_{\mu}(g) \neq 0\right\}$.

Clairement $\omega_{\mu}$ est un ouvert connexe et dense de $G_{\mathbf{C}}$.

(1.10) THÉORÈME. Soit $\mu$ un poids dominant d'une représentation de classe 1 ; alors $\omega \subset \omega_{\mu}$; de plus si $\mu$ est régulier, on $a \omega=\omega_{\mu}$.

Soit $g \in \omega$, c'est-à-dire $g=k a n$, avec $k \in K_{\mathbf{C}}, a \in A_{\mathbf{C}}, n \in N_{\mathbf{C}}$; alors $f_{\mu}(g)=$ $\chi_{\mu}(a)$; en effet les deux côtés de l'égalité sont des fonctions holomorphes sur $K^{\mathbf{C}} \times$ $A^{\mathbf{C}} \times N^{\mathbf{C}} ;$ si $g \in K A N$, alors

$$
\begin{aligned}
f_{\mu}(g) & =\left\langle\pi(k) \pi(a) \pi(n) e_{\mu}, e_{K}\right\rangle \\
& =\left\langle\pi(a) e_{\mu}, \pi\left(k^{-1}\right) e_{K}\right\rangle=\chi_{\mu}(a)\left\langle e_{\mu}, e_{K}\right\rangle=\chi_{\mu}(a)
\end{aligned}
$$

d'où l'égalité cherchée; par suite si $g \in \omega$, on a bien $f_{\mu}(g) \neq 0$.

Supposons maintenant $\mu$ régulier; comme $\omega \subset \omega_{\mu}$, et que $\omega_{\mu}$ est connexe, il suffit de montrer que $\omega$ est fermé relativement à $\omega_{\mu}$. Soit $g_{i}=k_{i} a_{i} n_{i}$ pour $i \geq 1$ une suite dans $\omega$, convergeant vers un point $g \in \omega_{\mu}$; soit $\gamma_{i}=\theta\left(g_{i}^{-1}\right) g_{i}$; alors $\gamma_{i}=\theta\left(n_{i}^{-1}\right) a_{i}^{2} n_{i}$, et donc $F_{\mu}\left(\gamma_{i}\right)=\chi_{\mu}\left(a_{i}^{2}\right)=f_{\mu}\left(g_{i}\right)^{2}$ tend vers $f_{\mu}(g)^{2} \neq 0$ puisque $g \in \omega_{\mu} ;$ maintenant

$$
\pi\left(\gamma_{i}\right) e_{\mu}=\pi\left(\theta\left(n_{i}^{-1}\right)\right) e_{\mu} \chi_{\mu}\left(a_{i}^{2}\right)
$$

ou encore

$$
\pi\left(\theta\left(n_{i}^{-1}\right)\right) e_{\mu}=f_{\mu}\left(g_{i}\right)^{-2} \pi\left(\gamma_{i}\right) e_{\mu} .
$$

La suite $\pi\left(\theta\left(n_{i}^{-1}\right)\right) e_{\mu}$ est donc convergente dans $E_{\mu}$; comme précédemment on en déduit d'après le lemme 1.7 que $\theta\left(n_{i}^{-1}\right)$ converge dans $\bar{N}_{\mathbf{C}}$, et donc $\left(n_{i}\right)$ converge vers $n$ dans $N_{\mathbf{C}}$.

On en déduit aussitôt que la suite $a_{i}^{2}$ converge vers un élément $b$ de $A_{\mathbf{C}}$; soit $a$ une racine carrée de $b$ (i.e. $a^{2}=b$ ); alors comme $F=K_{\mathbf{C}} \cap A_{\mathbf{C}}$ est fini, on voit facilement, quitte à modifier $a_{i}$ par un élément de $F$ (et $k_{i}$ en conséquence), qu'on peut supposer que $a_{i} \rightarrow a$; d'où $k_{i} \rightarrow k \in K^{\mathrm{C}}$, et $g=k a n \in \omega$.

Soit $G=K A N$ la décomposition classique d'Iwasawa, et notons $k(g), a(g)$ et $n(g)$ les trois composantes; ce sont des fonctions analytiques réelles sur $G$.

(1.11) THÉORÈME. (i) La fonction $n(g)$ se prolonge en une fonction analytique dans l'ouvert $\omega$, à valeurs dans $N_{\mathbf{C}}$.

(ii) Les fonctions $a(g)$ et $k(g)$ se prolongent en fonctions analytiques multivalentes dans l'ouvert $\omega$, à valeurs dans respectivement, $A_{\mathbf{C}}$ et $K_{\mathbf{C}}$; en un point $g_{0} \in \omega$, pour toute branche $a_{1}$ définie au voisinage de $g_{0}$, on peut trouver une unique branche $k_{1}$ de $k$ telle que $g=k_{1}(g) a_{1}(g) n(g)$. Si $a_{2}$ est une autre branche, et $k_{2}$ la branche correspondante, il existe $d \in F$, avec $k_{1}=k_{2} d$ et $a_{1}=a_{2} d=d a_{2}$.

DÉMONSTRATION. Si $g=k a n$, on a $\theta\left(g^{-1}\right) g=\theta\left(n^{-1}\right) a^{2} n$.

On déduit du théorème (1.8) que $n(g)$ et $a^{2}(g)$ s'étendent en fonctions analytiques dans $\omega$ tout entier. La partie (i) en résulte aussitôt. Ensuite comme $A^{\mathbf{C}}$ est isomorphe à $\left(\mathbf{C}^{*}\right)^{l}$, où $l$ est le rang de l'espace symétrique $G / K$ (ou $\left.U / K\right)$, on peut définir l'application $b \rightarrow b^{1 / 2}$ comme une fonction analytique multivalente; enfin $k(g) a(g)$ s'étend en une fonction holomorphe (univalente) puisque $k(g) a(g)=$ $g n^{-1}(g)$. Ceci, compte tenu de ce que $K_{\mathbf{C}} \cap A_{\mathbf{C}}=F$ termine la démonstration de (ii). 
Si $g \in \omega$, on désigne par $\mathcal{H}(g)$ la fonction holomorphe multivalente, à valeurs dans $\mathfrak{a}$, telle que exp $\mathcal{H}(g)=a(g)$.

REMARQUE. Le théorème (1.11) est fortement inspiré de la thèse de Van den Ban [13]; notre apport est la caractérisation de l'ouvert $\omega$ en termes plus intrinsèques (théorème 1.10); cette caractérisation est elle-même une variante d'un résultat d'Harish-Chandra [5, p. 285]; voir aussi [9].

2. Formule intégrale pour les fonctions sphériques de $U / K$. Avec les notations précédentes, introduisons la fonction sphérique associée à la représentation $\pi_{\mu}$, soit

$$
\varphi_{\mu}(g)=\left\langle\pi_{\mu}(g) e_{K}^{\prime}, e_{K}^{\prime}\right\rangle,
$$

où $e_{K}^{\prime}$ est un vecteur $K$-invariant de norme 1 (noter que $\varphi_{\mu}$ est indépendante du choix d'un vecteur particulier).

Comme $\int_{K} \pi_{\mu}(k) e_{\mu} d k$ est un vecteur $K$-invariant non nul, et que

$$
\left\langle\int_{K} \pi_{\mu}(k) e_{\mu} d k, e_{K}\right\rangle=\left\langle e_{\mu}, e_{K}\right\rangle=1,
$$

il vient

$$
\varphi_{\mu}(g)=\int_{K}\left\langle\pi_{\mu}(g k) e_{\mu}, e_{K}\right\rangle d k .
$$

(2.1) Lemme. Soit $g \in G_{\mathbf{C}}$; soit $K_{g}=\{k \in K, g k \in \omega\}$. Alors $K_{g}$ est un ouvert de $K$, dont le complémentaire est de mesure nulle.

Soit en effet $\mu \in \Lambda$, et considérons la fonction $k \rightarrow\left\langle\pi(g k) e_{\mu}, e_{K}\right\rangle$; Cette fonction n'est pas identiquement nulle sur $K$; en effet $\left\{\pi(k) e_{\mu}\right\}_{k \in K}$ est un système total dans $E_{\mu}$ (à cause de la décomposition d'Iwasawa de $G$, le sous-espace engendré coïncide avec celui engendré par $\left\{\pi(g) e_{\mu}\right\}_{g \in G}$, donc avec $E_{\mu}$ puisque $\pi$ est irréductible); par suite $e_{K}$ serait orthogonal à $E_{\mu}$ si la fonction considérée était identiquement nulle. Comme cette fonction est analytique réelle, le complémentaire de l'ensemble où elle s'annule set un ouvert dense, dont le complémentaire est de mesure nulle; on obtient le lemme grâce au théorème (1.10).

Si maintenant $k \in K_{g}$, alors $g k \in \omega$ a une décomposition de la forme $g k=$ $k(g k) a(g k) n(g k)$, et

$$
\left\langle\pi_{\mu}(g k) e_{\mu}, e_{K}\right\rangle=\chi_{\mu}(a(g k))=e^{\mu(\mathcal{H}(g k))} .
$$

On notera que le membre de droite ne dépend pas de la branche choisie, puisque $\chi_{\mu}$ vaut 1 sur $F$.

(2.2) THÉORÈME.

$$
\varphi_{\mu}(g)=\int_{K_{g}} e^{\mu(\mathcal{H}(g k))} d k \quad \forall g \in G_{\mathbf{C}} .
$$

Si $g \in G$, alors $K_{g}=K$, et on retrouve la formule classique d'Harish-Chandra (un cas particulier en fait, puisqu'alors le second membre est défini pour tout $\mu \in \mathfrak{a}_{\mathbf{C}}^{*}$ ).

REMARQUE. Qu'une telle formule existe pour $g$ voisin de $e K$ est élémentaire, et a déjà été utilisée par l'auteur [2] et d'autres $[10 ; 7$, p. 540].

Lorsque l'on se restreint à $U$, on a une propriété supplémentaire. 
(2.3) LEMME. $\left|\left\langle\pi(u) e_{\mu}, e_{K}\right\rangle\right| \leq 1$, pour $u \in U$.

Soit d'abord $g \in G$. Alors si $g=k a n$ est sa décomposition

$$
\left\langle\pi(g) e_{\mu}, e_{K}\right\rangle=\left\langle\pi(a) e_{\mu}, e_{K}\right\rangle=\chi_{\mu}(a) .
$$

Mais $\chi_{\mu}(a)=\left\|\pi(a) e_{\mu}\right\|=\left\|\pi(g) e_{\mu}\right\|$.

Soit $e_{1}=e_{\mu}, e_{2}, \ldots, e_{d}$ une base orthonormée de $E_{\mu}\left(d=\operatorname{dim}\left(E_{\mu}\right)\right)$, telle que chaque vecteur $e_{i}$ soit un vecteur de poids $\chi_{i}$ pour $A_{\mathbf{C}} ;$ alors

$$
\pi(k) e_{\mu}=\sum_{i=1}^{d} c_{i}(k) e_{i}
$$

et

$$
\pi(a) \pi(k) e_{\mu}=\sum_{i=1}^{d} c_{i}(k) \chi_{i}(a) e_{i} .
$$

Comme les $\chi_{i}(a)$ sont, pour $a \in A$, des réels positifs, on a

$$
\left\|\pi(a k) e_{\mu}\right\|^{2}=\sum_{i=1}^{d}\left|c_{i}(k)\right|^{2} \chi_{i}(a)^{2}
$$

avec

$$
\sum_{i=1}^{d}\left|c_{i}(k)\right|^{2}=1
$$

D'où finalement

$$
\left\langle\pi(a k) e_{\mu}, e_{K}\right\rangle^{2}=\sum_{i=1}^{d}\left|c_{i}(k)\right|^{2} \chi_{i}(a)^{2} .
$$

Cette égalité est valable pour $a \in A$; mais les deux membres sont des fonctions holomorphes sur $A_{\mathbf{C}}$, et donc l'égalité est encore valable pour $a \in A_{\mathbf{C}}$, en particulier pour $a \in \exp i$.

Si $u$ est un élément quelconque de $U$, alors $u=k_{1} a k_{2}$, avec $k_{1}, k_{2} \in K$, et $a \in \exp i \mathfrak{a} ;$ et $\left\langle\pi(u) e_{\mu}, e_{K}\right\rangle=\left\langle\pi\left(a k_{2}\right) e_{\mu}, e_{K}\right\rangle$. Par suite

$$
\left|\left\langle\pi(u) e_{\mu}, e_{K}\right\rangle\right|^{2} \leq \sum_{i=1}^{d}\left|c_{i}\left(k_{2}\right)\right|^{2}\left|\chi_{i}(a)\right|^{2}=1 .
$$

(2.4) Corollaire. Pour $u \in U, \operatorname{Re} \mu(H(u k)) \leq 0$.

Noter que cette expression ne dépend pas de la branche choisie pour $\mathcal{H}$, et que si $k \notin K_{u}$, on doit l'interpréter comme valant $-\infty$.

3. Equivalent asymptotique des fonctions sphériques de $U / K$. Soit $\mathfrak{a}_{r}=\{H \in \mathfrak{a} \mid \lambda(H) \notin \pi \mathbf{Z}, \forall \lambda \in \Sigma\}$, et soit $Q_{0}$ la composante connexe de $\mathfrak{a}_{r}$ contenue dans la chambre de Weyl positive $\mathfrak{a}^{+}=\left\{H \in \mathfrak{a} \mid \lambda(H)>0, \forall \lambda \in \Sigma_{+}\right\}$et contenant 0 dans son adhérence. Clairement pour $H \in Q_{0}$, on a $0<\lambda(H)<\pi$, pour tout $\lambda \in \Sigma^{+}$. 
(3.1) PROPOSITION. Tout élément $u$ appartenant à $U$ peut s'écrire $u=$ $k_{1} \exp i H k_{2}$, avec $H \in \bar{Q}_{0}$. De plus $u$ est régulier si et seulement si $H \in Q_{0}$ (cf. [6, p. 323]).

Par suite, on voit qu'une fonction sphérique, étant bi $K$-invariante, est déterminée par sa restriction à $\exp i \bar{Q}_{0}$.

On peut décrire $\Lambda$ à l'aide des "poids sphériques fondamentaux"; plus précisément, il existe (cf. $[\mathbf{1 1}, \mathbf{1 4}])$ des éléments $\mu_{1}, \mu_{2}, \ldots, \mu_{l}$ de $\Lambda$, avec $l=\operatorname{dim} \mathfrak{a}=$ $\operatorname{rang}(U / K)$, tel que les éléments de $\Lambda$ sont exactement les combinaisons linéaires à coefficients entiers positifs ou nuls des $\left\{\mu_{i}\right\}_{1 \leq i \leq l} ;$ si $\mu=\sum_{i=1}^{l} m_{i} \mu_{i}$, alors $\langle\mu, \mu\rangle^{1 / 2}$ est équivalent à $\sum_{i=1}^{l}\left|m_{i}\right|$, et $\mu$ est régulier si et seulement si $m_{i} \neq 0$ pour tout $i$, $1 \leq i \leq l$.

Dans ce paragraphe, nous allons faire tendre $\mu$ vers l'infini, en restant dans un cône à base compacte contenue dans le cône $\mathfrak{a}_{+}^{*}=\left\{\mu \in \mathfrak{a}^{*},\langle\mu, \lambda\rangle>0\right.$ pour $\left.\lambda \in \Sigma_{+}\right\}$.

En d'autres termes, nous supposerons que $\|\mu\|$ tend vers l'infini, et qu'il existe une constante $c>0$, avec $m_{i}(\mu)>c\|\mu\|, 1 \leq i \leq l$.

On se propose pour $a$ fixé et régulier (on peut en fait autoriser $a$ à varier dans un compact contenu dans l'ouvert des points réguliers), de déterminer un équivalent asymptotique de $\varphi_{\mu}(a)$ lorsque $\mu$ tend vers l'infini au sens précédent.

Avec les notations précédentes, on a la formule produit

$$
f_{\mu}=\prod_{i=1}^{l} f_{\mu_{1}}^{m_{1}} \cdots f_{\mu_{l}}^{m_{l}}, \quad 1 \leq i \leq l, \text { si } \mu=\sum_{i=1}^{l} m_{i} \mu_{i} .
$$

En effet, si $g \in G$, alors:

$$
\begin{aligned}
f_{\mu}(g) & =e^{\mu(\mathcal{H}(g))}=\left(e^{\mu_{1}(\mathcal{H}(g))}\right)^{m_{1}} \cdots\left(e^{\mu_{l}(\mathcal{H}(g))}\right)^{m_{l}} \\
& =\left(f_{\mu_{1}}\left(g_{1}\right)\right)^{m_{1}} \cdots\left(f_{\mu_{l}}\left(g_{l}\right)\right)^{m_{l}} .
\end{aligned}
$$

Comme les deux membres sont des fonctions holomorphes sur $G_{\mathbf{C}}$, on en déduit l'égalité annoncée.

On va profiter de cette remarque pour éliminer l'(éventuel) ensemble exceptionnel $K \backslash K_{a}$. Soit en effet $\mu \in \Lambda$; on a donc $\mu=\sum_{i=1}^{l} m_{i} \mu_{i}$; soit $\tilde{K}_{a}=\{k \in$ $\left.K,\left|f_{\mu_{i}}(a k)\right| \geq \frac{1}{2}, \forall i, 1 \leq i \leq l\right\}$; alors si $k \in K \backslash \tilde{K}_{a}$, on a, pour au moins une valeur de $i,\left|f_{\mu}(a k)\right| \leq\left(\frac{1}{2}\right)^{m_{i}}$.

Par suite dans la recherche d'un équivalent asymptotique de $\varphi_{\mu}(a)$ lorsque $\mu$ tend vers l'infini, on peut négliger la contribution du complémentaire de $\tilde{K}_{a}$ dans l'intégrale $\int_{K} f_{\mu}(a k) d k$, l'erreur étant $O\left(\|\mu\|^{-N}\right)$ pour tout $N$.

Maintenant, sur $\tilde{K}_{a}$ on peut utiliser l'extension analytique multiforme de la fonction $\mathcal{H}$; au moyen d'un recouvrement fini et d'une partition de l'identité subordonnée, on peut supposer qu'on a choisi localement une détermination de la fonction H.

L'intégrale qui exprime $\varphi_{\mu}$ peut alors s'étudier à l'aide de la méthode de la phase stationnaire complexe; la condition $\operatorname{Re} \mu(\not H(a k)) \leq 0$ établie à la fin du paragraphe précédent est exactement ce qui est exigé pour pouvoir la mettre en oeuvre (cf. [8, 1]). 
Il nous faut donc calculer les points critiques de la fonction de phase; pour $a \in \exp i \mathfrak{a}$, et $\mu \in \Lambda$, posons

$$
\psi_{a, \mu}(\dot{k})=\langle\mu, \mathcal{H}(a k)\rangle,
$$

considérée comme unc fonction définie sur $K / M$ (localement).

(3.2) Proposition. Soit $\mu \in \Lambda$ régulier et a régulier; alors les points critiques de la fonction $\psi_{a, \mu}$ sont exactement les classes $w M$, où $w$ parcourt le groupe de Weyl $W=N_{K}(T) / T$.

Cette proposition résulte, par prolongement analytique de calcul de la différentielle de $\psi_{a, \mu}$ effectué en [4].

Posons $a=\exp i H$, avec $H \in Q_{0}$; on a comme détermination possible de $H(a w)=H\left(w^{-1} a w\right)=i \operatorname{Ad} w^{-1} \cdot H=i w^{-1} \cdot H$.

Il faut maintenant déterminer la forme hessienne de $\psi_{a, \mu}$ en un tel point critique. Nous suivons à nouveau [4]. D'abord quelques notations supplémentaires. Soit $\mathfrak{l}=\mathfrak{k} \ominus \mathfrak{m}$ l'orthogonal de $\mathfrak{m}$ dans $\mathfrak{k} ; \mathfrak{l}$ s'identifie au plan tangent en $a^{w^{-1}}$ à $K / M$. Pour $\lambda \in \Sigma_{+}$, soit $\mathfrak{l}_{\lambda}=\left(\mathfrak{g}_{\lambda}+\theta \mathfrak{g}_{\lambda}\right) \cap \mathfrak{k}$.

On a $\mathfrak{l}=\bigoplus_{\lambda \in \Sigma_{+}} \mathfrak{l}_{\lambda}$; désignons par $F_{\lambda}$ le projecteur orthogonal de $\mathfrak{l}$ sur $\mathfrak{l}_{\lambda}$. Soit enfin $($, ) le produit scalaire euclidien sur l égal à l'opposé de la forme de Killing.

(3.3) Proposition. Soit $\mu$ et a comme en (3.2), et $w \in W$. Alors la forme hessienne de $\psi_{a, \mu}$ en $w M$ est donnée par

$$
d^{2} \psi_{a, \mu}(Y, Z)=\left(Y, L_{a, \mu, w}(Z)\right), \quad Y, Z \in \mathfrak{l}
$$

avec

$$
L_{a, \mu, w}=-\frac{1}{2} \sum_{\lambda \in \Sigma^{+}}\langle\lambda, \mu\rangle\left(1-e^{-2 w \lambda(i H)}\right) F_{\lambda} .
$$

Si $\operatorname{Re} z \geq 0$, notons $z^{1 / 2}$ l'unique élément $\rho \in C$ tel que $\rho^{2}=z$, et $\operatorname{Re} \rho \geq 0$. Posant pour un instant $Q=-L_{a, \mu, w}$, on doit calculer $(\operatorname{det} Q)^{1 / 2}$, où l'on choisit la détermination de la racine carrée qui est continûment déformée en 1 par l'homotopie $[0,1] \ni s \rightarrow s I+(1-s) Q$.

Mais $Q$, d'après (3.3) est donnée sous forme diagonale. Par suite, on peut définir $Q^{1 / 2}$ sans difficulté.

Si $w \lambda \in \Sigma_{+}$, alors on écrit

$$
\frac{1}{2}\left(1-e^{-2 w \lambda(i H)}\right)=i e^{-i w \lambda(H)} \sin w \lambda(H),
$$

et comme $0<w \lambda(H)<\pi$, on doit prendre comme racine carrée

$$
e^{i \pi / 4} e^{-i w \lambda(H) / 2}|\sin w \lambda(H)|^{1 / 2} .
$$

Si au contraire $w \lambda \in-\Sigma_{+}$, alors on écrit

$$
\frac{1}{2}\left(1-e^{-2 w \lambda(i H)}\right)=-i e^{-i w \lambda(H)}|\sin w \lambda(H)|,
$$

et on prend comme racine carrée

$$
e^{-i \pi / 4} e^{-i w \lambda(H) / 2}|\sin w \lambda(H)|^{1 / 2} .
$$


Pour énoncer le résultat final, introduisons pour $w \in W$

$$
\gamma(w)=\sum_{\substack{\lambda \in \Sigma_{+} \\ w \lambda \in \Sigma_{+}}} m_{\lambda}-\sum_{\substack{\lambda \in \Sigma^{+} \\ w \lambda \in-\Sigma^{+}}} m_{\lambda},
$$

où $m_{\lambda}=\operatorname{dim} g_{\lambda}$ désigne la multiplicité de la racine restreinte $\lambda$, et soit comme d'habitude $\rho=\frac{1}{2} \sum m_{\lambda} \lambda$ la demi-somme des racines positives restreintes. Enfin on note $\operatorname{vol}(K / M)$ le volume de $K / M$ pour la métrique riemannienne associée au produit scalaire $($,$) (opposé de la forme de Killing) sur l.$

(3.4) THÉORÈme. Soit $H \in Q_{0}$ et $a=\exp i H$; alors

$$
\begin{aligned}
\varphi_{\mu}(a) \sim \operatorname{vol}(K / M)^{-1}(2 \pi)^{d / 2} \prod_{\lambda \in \Sigma^{+}}\langle\lambda, \mu\rangle^{-m_{\lambda} / 2} & \prod_{\lambda \in \Sigma^{+}} \sin \lambda(H)^{-m_{\lambda} / 2} \\
& \cdots \sum_{w \in W} e^{-i \gamma(w) \pi / 4} e^{i\langle w(\mu+\rho), H\rangle},
\end{aligned}
$$

lorsque $\mu$ tend vers l'infini en restant dans un cône à base compacte contenue dans $\mathfrak{a}_{+}^{*}$.

Plus précisément, la différence entre les deux expressions est $O\left(\|\mu\|^{-d / 2-1}\right)$, où $d=\sum_{\lambda \in \Sigma_{+}} m_{\lambda}=\operatorname{dim} K / M$ est la dimension d'une orbite générique de $K$ dans $U / K$. De plus cette estimation est localement uniforme lorsque $H$ varie en restant dans $Q_{0}$.

Moyennant les calculs précédents, ceci est l'application du résultat de [8]. Le coefficient $\operatorname{vol}(K / M)^{-1}$ s'introduit, car la mesure choisie sur $K / M$ est de masse 1 , alors que la mesure riemannienne à utiliser est obtenue à partir du produit scalaire (,) (opposé de la forme de Killing) sur l.

\section{REFERENCES}

1. D. Barlet and J. L. Clerc, Le comportement à l'infini des fonctions de Bessel généralisées. I, Adv. in Math. (à paraître).

2. J. L. Clerc, Une formule asymptotique de type Melher-Heine pour les zonales d'un espace riemannien symétrique, Studia Math. 57 (1976), 27-32.

3. __ Transformées de Fourier des mesures orbitales dans l'espace tangent d'un espace symétrique (à paraître).

4. J. J. Duistermaat, J. A. C. Kolk and V. S. Varadarajan, Functions, flows and oscillatory integrals on flag manifolds and conjugacy classes in real semi simple Lie groups, Compositio Math. 49 (1983), 309-398.

5. Harish-Chandra, Spherical functions on a semi-simple Lie group. I, Amer. J. Math. 80 (1958), 241-310.

6. S. Helgason, Differential geometry, Lie groups and symmetric spaces, Academic Press, New York, 1978.

7. __ Groups and geometric analysis, Academic Press, New York, 1984.

8. A. Melin and J. Sjöstrand, Fourier integral operators with complex valued phase functions, Lecture Notes in Math., vol. 459, Springer-Verlag, pp. 120-223.

9. T. O. Sherman, Fourier analysis on compact symmetric space, Bull. Amer. Math. Soc. 83 (1977), 378-380.

10. R. J. Stanton, On mean convergence of Fourier series on compact Lie groups, Trans. Amer. Math. Soc. 218 (1976), 61-87.

11. M. Sugiura, Representations of compact groups realized by spherical functions on symmetric spaces, Proc. Japan Acad. 38 (1962), 111-113. 
12. G. Szegö, Orthogonal polynomials, Amer. Math. Soc. Colloq. Publ., vol. 23, Amer. Math. Soc., Providence, R. I., 1939.

13. E. P. Van den Ban, Asymptotic expansions and integral formulas for eigenfunctions on a semisimple Lie groups, Thesis, Utrecht, 1983.

14. L. Vretare, Elementary spherical functions on symmetric spaces, Math. Scand. 39 (1976), 343358.

Département de Mathématiques, U.A. 750, Faculté des Sciences, B.P. 239, 54506-VANDOEUVRE-LES-NANCY, FRANCE 